\title{
Outward transmission capacity analysis under the background of UHV AC/DC power grid
}

\author{
Yang Liu ${ }^{1, a}$, Jianhua Zhang ${ }^{1}$, Rishang Long ${ }^{1}$, Yuan Meng ${ }^{1}$, Hao Hu ${ }^{1}$, Tao Tan \\ 1, Mingxin $\mathrm{Xu}^{2, \mathrm{a}}$, Long Zhao ${ }^{2, \mathrm{a}}$ \\ ${ }^{1}$ State Key Laboratory of new energy power system, North China Electric Power University, Beijing, \\ China,102206 \\ ${ }^{2}$ Inner Mongolia Eastern Electric Power Co., Ltd. Economic and Technical Research Institute, Inner \\ Mongolia, China, 010010 \\ aliuyangyx1003@163.com
}

Keywords: DC power transmission, multi-send short circuit ratio, outward transmission capacity, stability analysis

Abstract. Considering the stability analysis theory of multi-infeed high voltage direct current (HVDC) system, the transmission capacity analysis method of multi-send ultra high voltage (UHV) AC/DC power grid is studied in this paper. By extending the multi-infeed short circuit ratio index, we can get the multi-send short circuit ratio index (MSSCR), and the stability of the system is analyzed by using BPA simulation platform. Then MSSCR is revised according to the analysis results, and adjust the outward transmission capacity until MSSCR reached set value, which is the outward transmission capacity of the grid.

\section{Introduction}

China has a vast territory and abundant energy, but the distribution of the load center and the power center is not balanced. The power demand will increase with the economic development and due to the contradiction between the load and power distribution in China, a large-scale power flow configuration of the whole country will form in the future [1]. With the policy formulation about the electric energy and the implementation of "power transmission from West to East" and "power exchange between North and South", the intensity of national energy resource optimization configuration will further increase, so AC and DC coupling between the regional power grids will be stronger [2-4].

With the continuous construction of DC transmission lines, grid structure is more and more complex. The grid connection mode changes from the simple single DC connection of two non-synchronous power grids to the large-scale AC / DC hybrid connection, and the grid connection scale changes from simple single DC connection to the large-scale AC/DC connection. These changes ease the contradictions and problems caused by uneven distribution of the resource and load, but with the power grid structure is more and more complex, there will be more and more problems about the security and stability of power grid.

But at the moment, most studies about AC/DC hybrid system are more focused on the stability of the multi-infeed HVDC system [5-8], and ignore the relevant circumstances of multi-send HVDC system. Reference [9] analyzes the operation condition of Ya' an-Jinmen $100 \mathrm{kV}$ UHV line by using the quantitative evaluation index and small signal stability analysis method, and gets the influence to other three DC lines. But quantitative evaluation indexes used in reference [9] are all about the multi-infeed HVDC system, such as multi-infeed interaction factor and multi-infeed short circuit ratio, and the indexes about multi-send HVDC system are not put forward. Reference [10] studies the coordinated control operation and equipment operating characteristics of UHV DC system including wind power, and puts forward the active power control strategy of DC power coordinated control following wind power fluctuations, which can improve the fault recovery ability of the system, but its transmission capacity is not analyzed specifically. However, in fact the sending-end system tends to be an area where many energy sources are relatively concentrated, and the coupling relationship between each sending lines is relatively close (including DC and AC sending lines) [11]. If the space truss structure of the 
sending-end system is weak, the influence between AC and DC lines or each DC lines in the sending-end system cannot be ignored.

This paper analyzes the development actuality of UHV AV/DV hybrid power grid at home and abroad, extends the analysis method of the power receiving capability of the multi-infeed DC system, and puts forward multi-send short circuit ratio index and the analysis method of the outward transmission capacity of the AC power grid, and the validity of this method is proved through the actual example.

\section{Sending form of the UHV AC / DC hybrid power grid}

In the AC / DC hybrid power grid, there are AC transmission lines firstly, and in order to enhance the external outward transmission capacity of the system, some DC transmission lines are constructed [12]. According to the different structure, the sending form of the sending-end system can be divided into three kinds: send power from power plant directly, send power from single point within the network and send power from more points within the network, as shown in Figure 1.

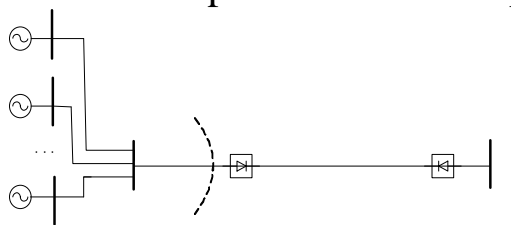

(a)

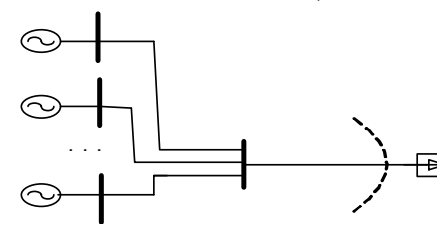

Send power from power plant directly point within the network

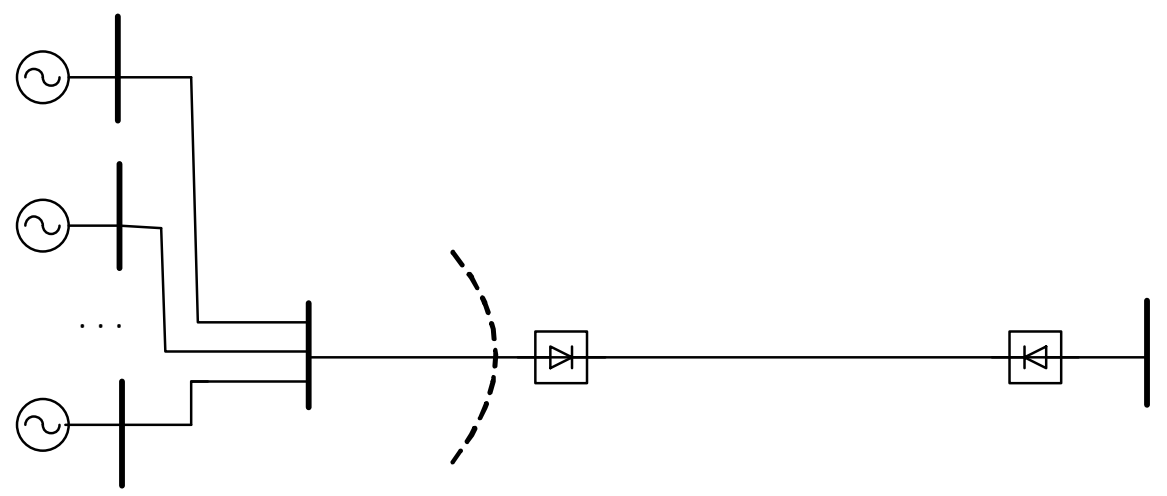

(c)

Send power from more points within the network

Figure 1. The sending form of the sending-end system

Among them, the method of sending power from power plant directly is applicable for the large capacity power plant to deliver the power to the load center. The method of sending power from single point within the network is similar to the first one, but takes the influence of the DC transmission lines into consideration. The fault in the AC system may lead to the voltage fluctuation of the rectifier bus, and if the fluctuation is large enough, it will result in the failure of the DC transmission line and losing the transmission capacity. The method of sending power from more points within the network is to deliver the power to the outside network by multiple DC transmission lines, which is suitable for the wind farm interconnection or a variety of new energy power generations interconnected. It can improve the flexibility and diversity of power supply effectively.

\section{Outward transmission capacity analysis index}

\section{DC mutual influence factor (DMIF)}

In the methods of sending power from power plant directly and sending power from single point within the network, there is only one DC transmission line, so there is no DMIF; in the method of sending power from more points within the network, there may be several DC transmission lines, so we need to study the strength of influence between the each DC lines. DMIF can be defined as the droop 
rate of voltage of the $\mathrm{DC}$ line $\mathrm{j}$, when the voltage of the rectifier bus $\mathrm{i}$ declines $1 \%$ under the disturbance, and its formula is:

$$
D M I F_{j i}=\frac{\Delta U_{j}}{\Delta U_{i}} .
$$

Where $\Delta U_{i}$ is the droop rate of voltage of the rectifier bus $i$ after adding a parallel reactive power branch to it, and its value is $1 \% ; \Delta U_{j}$ is the droop rate of voltage of the DC line $j$.

\section{Multi-send short circuit ratio (MSSCR)}

In the method of sending power from more points within the network, there is the influence between $\mathrm{AC}$ and DC, which depends on the relative capacity of the DC system and the AC system. In order to evaluate the mutual influence between the DC and AC lines in the multi-send DC system, extend the traditional short circuit and multi-infeed short circuit ratio, put forward the multi-send short circuit ratio, and its formula is:

$$
M S C R_{i}=\frac{S_{a c i}}{P_{d i}+\sum_{j=1, j \neq i}^{K} D M I F_{j i} \cdot P_{d j}}=\frac{S_{a c i}}{P_{d i}+\sum_{j=1, j \neq i}^{K} \frac{\Delta U_{j}}{\Delta U_{i}} \cdot P_{d j}} .
$$

Where $M S C R_{i}$ is multi-infeed short circuit ratio of the DC line $i, S_{a c i}$ is short circuit capacity of the rectifier side bus of the DC line $i, P_{d i}$ and $P_{d j}$ are active power transferred by the DC line $i$ and $j$, respectively. $D M I F_{j i}$ is the DC mutual influence factor.

According to the previous research on multi-infeed short circuit ratio, we can know that if multi-send short circuit ratio is larger, the intensity of the system is stronger.

\section{The analysis method of the outward transmission capacity}

Combining the indexes described in the second II, analyze the outward transmission capacity, and the specific idea is: first, set up the system model, including grid contact lines, as the basis of analysis of the outward transmission capacity; Then, according to the different strength requirement of the power grid, set the value of MSSCR; according to the transmission mode and assumed outward transmission power value, calculate MSSCR and determine whether the results meet the requirements. If meet, the outward transmission power value is the outward transmission capacity of the grid, otherwise, it is needed to constantly adjust the outward transmission power value and recalculate MSSCR until it can meet the set value. Then we can choose the corresponding fault according to the principle of fault selection for stability analysis. If the electric grid can't remain stable, reset the MSSCR and continue calculating until the grid is stable.

The flow chart of the outward transmission capacity analysis method is shown in Figure 2. 


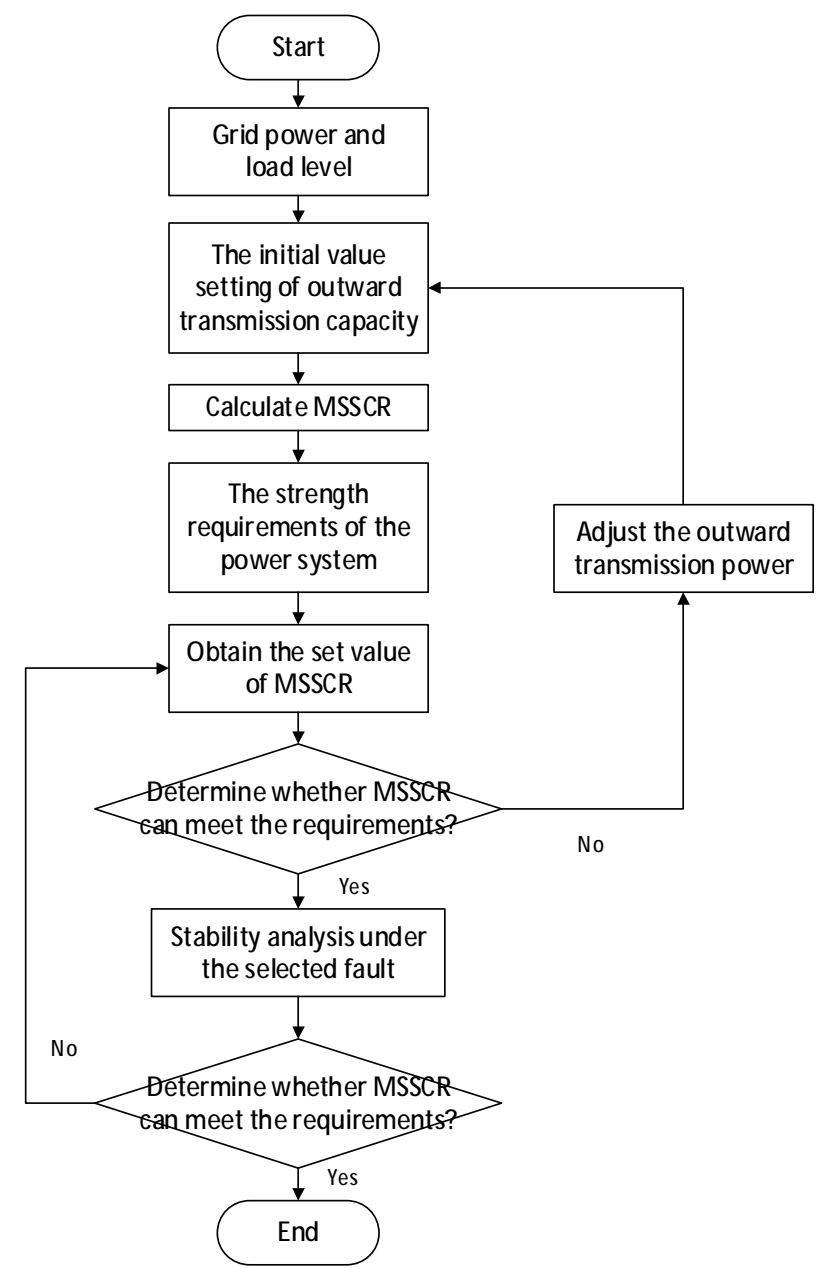

Figure 2. The flow chart of the outward transmission capacity analysis method

\section{Case study}

With the northwest power grid as an example for analysis, the northwest region has plenty of natural resources, including coal, oil natural gas and other mineral energy, so the generating capacity is very large. But its local load is far less than its generating capacity, therefore the northwest power will transfer power by UHVDC lines to other load center. Haminan-Zhengzhou UHVDC line has been built, and its capacity is 8 million $\mathrm{kW}$. And Jiuquan-Xiangtan UHVDC transmission project is planning to be built, in this paper, we are planning the transmission capacity of it.

According to the strength requirement of the power grid in the northwest region, the value of MSSCR is set to be 2.5. Calculate MSSCR under this outward power transmission method. At this time, the results of the calculation are shown in Table 1.

Table 1. The primary analysis results of the outward transmission capacity of the Northwest Power Grid

\begin{tabular}{ccc}
\hline Index & Haminan-Zhengzhou & Jiuquan-Xiangtan \\
\hline MSSCR & 2.50 & 4.54 \\
\hline
\end{tabular}

At this point, the outward transmission capacity is about 9 million $\mathrm{kW}$. Analyze the stability of the system, through the analysis, $1000 \mathrm{kV}, 500 \mathrm{kV}$ lines and all the transformer are not overloaded in the $\mathrm{N}-1$ fault, satisfying the static safety N-1standard. And in DC monopoles blocking faults, the stability of the system also meets the requirement. Because the position of the power sources of Jiuquan-Xiangtan and Hami-Zhengzhou are close, if there is a strong wind, earthquake and other serious disasters, the two DC lines may fault at the same time. So analyze the stability of the system under the condition of losing the UHVDC of the northwest thermal power at the same time, the results are shown in Table 2. 
Table 2. The voltage minimum value while losing UHVDC at the same time in the primary calculation

\begin{tabular}{ccc}
\hline Sequence number & The name of the bus & The voltage minimum value \\
\hline 1 & Guomichang & 0.74894 \\
2 & Guomihuan & 0.80501 \\
\hline
\end{tabular}

According to Table 2, the outward transmission capacity of Jiuquan-Xiangtan DC line is 9 million $\mathrm{kW}$. The impact to the Hami area is obvious, making its voltage below the standard of the electric power system. Thus if the strength of the sending-end system is too low, reset MSSCR and recalculate the results.

In order to improve the strength of grid, MSSCR is reset to be 3.0, and recalculate corresponding results under this outward power transmission method. The calculation results are shown in table 3 and table 4.

Table 3. The second analysis results of the outward transmission capacity of the Northwest Power Grid

\begin{tabular}{ccc}
\hline Index & Haminan-Zhengzhou & Jiuquan-Xiangtan \\
\hline MSSCR & 3.0 & 5.09 \\
\hline
\end{tabular}

Table 4. The voltage minimum value while losing UHVDC at the same time in the second calculation

\begin{tabular}{ccc}
\hline Sequence number & The name of the bus & The voltage minimum value \\
\hline 1 & Guomichang & 0.76561 \\
2 & Guomihuan & 0.81641 \\
\hline
\end{tabular}

At this point, the outward transmission capacity is about 8.5 million $\mathrm{kW}$. Analyze the stability of the system, through the analysis, $1000 \mathrm{kV}, 500 \mathrm{kV}$ lines and all the transformer are not overloaded in the $\mathrm{N}-1$ fault, satisfying the static safety N-1standard. And in DC monopoles blocking faults, the stability of the system also meets the requirement. According to Table 4, if the outward transmission capacity of Jiuquan-Xiangtan DC line is 8.5 million $\mathrm{kW}$, although still has not reached the requirements of power system, but the system voltage stability has been improved.

Repeat the process above, change the value of MSSCR constantly, and we can obtain the outward transmission capacity finally which can satisfy the requirement of system stability. This capacity is outward transmission capacity of the power grid.

\section{Conclusions}

In this paper, according to the stability analysis theory of multi-infeed DC system, outward transmission capacity analysis method of multi-send UHV AC/DC power grid. Revise the MSSCR on the basis of stability analysis results unceasingly, and adjust the outward transmission capacity until MSSCR reached the set value. In the process of analysis, can draw the following conclusion:

(1) According to the multi-infeed short circuit ratio index, we can put forward the multi-send short circuit ratio index to evaluate the mutual influence between AC/DC lines and each DC lines.

(2) With the increase of the outward transmission capacity of DC lines, the influence on the stability of power grid is greater in DC monopoles blocking faults. So revise MSSCR index according to the stability of power grid, then change the power of the outward transmission line, and finally obtain the outward transmission capacity of the power grid.

\section{Acknowledgements}

This work was financially supported by National Natural Science Foundation (51277067) and Central University Foundation (2015XS03). 


\section{References}

[1] Li Jing, Kuang Mingsheng. Energy Characteristics and Sustainable Development Strategy of China. Scientific and Technological Management of Land and Resources, 2009, 26(4): 60-64.

[2] Zhou Xiaoqian. Development, planning and implementation of the project of power transmission from West China to East China. Power System Technology, 2003, 27(5): 1-6.

[3] Zhang Jinhua, Wu Ziping, Hu Tao, Zhang Wenchao. Research on Coordinated Control Technology for AC/DC hybrid system in China. 2014 IEEE PES T\&D Conference and Exposition, 2014: 1-5.

[4] Zhou Jun, Guo Jianbo, Guo Qiang, Zhu Yiying. Security and stability study of planning UHVAV/DC HYBRID Transmission system. Electric Utility Deregulation and Restructuring and Power Technologies (DRPT), 2011 4th International Conference on,2011:1026-1030.

[5] Li Zhaowei, Zhai Haibao, Liu Fusuo, et al. DC access capability study for multi-infeed HVDC power transmission system. Power System Protection and Control, 2016, 44(8): 142-148.

[6] Tang Yi, Chen Bin, Pi Jingchuang, et al. Analysis on Absorbing Ability of Receiving AC System for UHVDC Hierarchical Connection to AV Grid. Proceedings of the CSEE, 2016, 36(7): 1790-1800.

[7] Yang Di, Cheng Haozhong, Yao Liangzhong, et al. Research Review on AC/DC Hybrid System With Multi-Terminal HVDV. Power System Technology, 2015, 39(8): 2201-2209.

[8] Pengfei Wang, Yingmin Zhang, Hu Chen, et al. Analysis on the Interaction of AC/DC System Based on Multi-Infeed Q Effective Short Circuit Ratio. 2012 Asia-Pacific Power and Energy Engineering Conference, 2012: 1-4.

[9] Chen Hu, Zhang Yingmin, He Yang, et al. Impact assessment of UHVAC on multi-send HVDC system of Sichuan power grid. Power System Protection and Control, 2011, 39(7): 136-141.

[10] Zhu Yiying, Dong Peng, Xie Guoping, et al. Real-Time Simulation of UHVDC Cooperative Control Suitable to Large-Scale Wind Farms. Power System Technology, 2013, 37(7): 1814-1819.

[11] Jingzhe Tu, Jian Zhang, Guangquan Bu, et al. Analysis of the Sending-Side System Instability Caused by Multiple HVDC Commutation Failure. CSEE JOURNAL OF POWER AND ENERGY SYSTEMS, 2015,1(4):37-44.

[12] Chen Kai, Duan Xiangying, Guo Xiaojiang, et al. Stability Control Analysis of UHV AC/DC Hybrid Power Grid. Electric Power Construction, 2016, 37(1): 64-69. 